\title{
El desempeño del sector de software y servicios informáticos en la Argentina: evidencia microeconométrica sobre los programas públicos de apoyo
}

\author{
Mariano Pereira, María Florencia Barletta y Gabriel Yoguel
}

\section{Resumen}

En el presente artículo se analiza el impacto que han tenido los programas públicos de apoyo en el desempeño reciente del sector de software y servicios informáticos de la Argentina. En primer lugar, se estudia su efecto en el desempeño innovador de las firmas. Se utiliza una técnica de emparejamiento por puntaje de propensión (propensity score matching) para calcular el efecto medio del tratamiento en las firmas beneficiarias. Los resultados confirman que la recepción de fondos públicos tuvo un impacto positivo en el ratio entre el gasto en investigación y desarrollo (I+D) y las ventas, el empleo en I+D y la propensión a introducir nuevos productos o procesos. En segundo lugar, se analiza el efecto de la intervención de política sobre el desempeño económico de las empresas. En este caso, se utilizó un diseño de variables instrumentales, y los resultados muestran un impacto positivo en la propensión exportadora, la intensidad de las exportaciones y el crecimiento del empleo.

Palabras clave

Informática, programas de computadora, industria, innovaciones, investigación y desarrollo, política de ciencia y tecnología, promoción industrial, modelos econométricos, Argentina

Clasificación JEL

C210, O320, L860

Autores

Mariano Pereira es Coordinador de la Unidad de Información, Monitoreo y Evaluación (UIME) del Centro Interdisciplinario de Estudios en Ciencia, Tecnología e Innovación (CIECTI) e Investigador Docente del Instituto de Industria de la Universidad Nacional de General Sarmiento (UNGS), Argentina. lic.mpereira@gmail.com

María Florencia Barletta es Investigadora Docente del Instituto de Industria de la Universidad Nacional de General Sarmiento, Argentina. fbarlett@ungs.edu.ar

Gabriel Yoguel es Investigador Docente del Instituto de Industria de la Universidad Nacional de General Sarmiento, Argentina. gyoguel@ungs.edu.ar 


\section{Introducción}

A comienzos de la década de 2000, los pronósticos sobre la dinámica del sector de software y servicios informáticos en la Argentina eran extremamente negativos. Nadie preveía que algo más de una década después el empleo en el sector iba superar el empleo en el complejo automotriz (un 8\% más), en diversos sectores primarios (servicios agrícolas), industriales (bienes de capital, cuero y calzado, madera, muebles, siderurgia) y de servicios (electricidad, gas y agua) y que habría casi alcanzado el mismo nivel de empleo de sectores que tenían un quántum notablemente superior a fines de los años noventa (bancos, seguros y servicios inmobiliarios). En diversos trabajos publicados se ponía de manifiesto que los problemas que enfrentaba el sector limitaban su sendero evolutivo. Esas restricciones se centraban en tres aspectos: i) capacidades comerciales excesivamente focalizadas en el mercado interno, que bloqueaban la posibilidad de desarrollar una industria competitiva en los mercados externos (López y Ramos, 2008); ii) débil desarrollo de las capacidades tecnológicas de los trabajadores, que condicionaba el grado de complejidad de los productos y servicios ofertados (Borello y otros, 2005), y iii) ausencia de una masa crítica de empresas de excelencia que desarrollaran productos propios, que limitaba la posibilidad de identificar perfiles sectoriales exitosos (Chudnovsky, López y Melitsko, 2001; López, 2003; Perazzo y otros, 1999). Sin embargo, a pesar de estas restricciones, el sector tomó un sendero muy distinto. En efecto, desde la devaluación del peso argentino en 2002 las empresas de software y servicios informáticos protagonizaron un crecimiento vertiginoso, con una dinámica agregada caracterizada por incrementos en el empleo, las ventas y las exportaciones muy superiores a los registrados en la industria manufacturera (Barletta y otros, 2013; Maldonado, Morero y Borrastero, 2013).

Entre los factores que permitirían explicar este desempeño se destacan: la mayor competitividad de la economía argentina a partir de la devaluación de principios de 2002, el aumento de la externalización en el desarrollo de software a nivel global (que permitió dinamizar un segmento importante de las empresas de software y servicios informáticos) y otras características culturales y contextuales favorables a la inserción externa (dominio del idioma inglés, husos horarios y disponibilidad de recursos humanos calificados, entre otros). Estos factores se complementaron con otros de naturaleza interna, como el fuerte crecimiento del mercado interno y el alto nivel de capacidades generadas en décadas previas en las empresas más antiguas y en la gran mayoría de las nuevas desde comienzos de la década de 2000, y con una amplia batería de programas públicos orientados a promover la certificación de calidad, las exportaciones y las actividades de investigación y desarrollo $(l+D)$, que impulsaron los esfuerzos de innovación. En los últimos años, los factores que determinaron el desempeño reciente del sector de software y servicios informáticos en la Argentina han sido objeto de diversos estudios. Sin embargo, son sorprendentemente pocos los trabajos que analizan los efectos de los programas de apoyo a la innovación en el desempeño del sector de servicios intensivos en conocimiento en general y de software y servicios informáticos en particular (Castro y Jorrat, 2013).

Con el presente artículo se busca alcanzar tres objetivos específicos, a saber: i) evaluar el efecto causal de los programas de apoyo a la innovación en el desempeño innovador y económico de las empresas; ii) analizar la existencia de derrames o efectos indirectos mediante los vínculos entre empresas beneficiarias y no beneficiarias para la realización de actividades conjuntas de I+D, y iii) evaluar la complementariedad entre instrumentos, pues las empresas pueden solicitar y recibir apoyo de más de un programa.

De acuerdo con estos objetivos, los interrogantes a los que se busca responder son: ¿qué papel desempeñaron los programas públicos de apoyo a la innovación en la conducta innovadora de las empresas de software y servicios informáticos?, ¿es posible afirmar que, a partir de esa conducta innovadora más virtuosa, los instrumentos de promoción del sector ayudaron a mejorar el rendimiento 
económico de las empresas? En definitiva, ¿qué papel desempeñó la política pública de ciencia y tecnología en el ponderable desempeño del sector durante la última década? Para dar respuesta a estas preguntas se utiliza una base de datos compuesta por 187 empresas de software y servicios informáticos, construida especialmente para captar las especificidades del sector. Sobre esa base se aplican dos técnicas de identificación para captar el efecto medio del tratamiento en los tratados en las empresas que recibieron subsidios, incentivos fiscales o ambos en 2010: i) emparejamiento por puntaje de propensión (Rosenbaum y Rubin, 1983), y ii) estimaciones doblemente robustas (doubly robust estimations) (Tsiatis, 2006; Leon, Tsiatis y Davidian, 2003; y Lunceford y Davidian, 2004).

El artículo se divide en cinco secciones, incluida esta Introducción. En la segunda se presentan la base de datos y la estadística descriptiva sobre las diferencias entre empresas beneficiarias y no beneficiarias. En la tercera sección se describe la propuesta metodológica para captar el efecto causal de la recepción de un beneficio público y en la cuarta se estima el efecto causal de participar en un programa público en la conducta innovadora y el desempeño económico de la empresa. La quinta y última sección contiene las principales conclusiones del artículo.

\section{Base de datos}

La base de datos utilizada en este artículo surge de una encuesta a empresas del sector de software y servicios informáticos del año 2011. El trabajo contó con el financiamiento de la Fundación Carolina de España y la ayuda del Observatorio de Empleo y Dinámica Empresarial del Ministerio de Trabajo, Empleo y Seguridad Social para el diseño de la muestra. Esta comprende un total de 189 empresas, 2 de las cuales no se tuvieron en cuenta debido al carácter incompleto de los datos. En promedio, las empresas examinadas empleaban alrededor de 60 personas cada una y declaraban ventas anuales por alrededor de ocho millones de pesos en el año 2010. El 56\% declaró tener alguna participación en el comercio exterior. La mayoría de las empresas de la muestra iniciaron sus actividades a finales de la década de 1990, se destacan por ser de origen nacional (93\%) y operan de forma independiente, ya que solo el $10 \%$ forma parte de un grupo empresarial (véase el cuadro 1).

Cuadro 1

Argentina: estadísticas descriptivas de las empresas de software y servicios informáticos, 2010

\begin{tabular}{lccccr}
\hline & Observaciones & Media & Desviación estándar & Mínimo & Máximo \\
\hline Empleo (en número de empleados) & 187 & 58 & 14 & 1 & 1500 \\
\hline Ventas (en millones de pesos) & 187 & 7,9 & 22,7 & 0 & 200 \\
\hline Exportaciones (0 = no exporta; 1 = exporta) & 187 & 0,56 & 0,5 & 0 & 1 \\
\hline Año de inicio & 187 & 1999 & 7 & 1968 & 2010 \\
\hline $\begin{array}{l}\text { Participación en grupo empresarial } \\
(0=\text { no participa; } 1 \text { = participa) }\end{array}$ & 187 & 0,1 & 0,3 & 0 & 1 \\
Capital extranjero (en porcentajes) & 187 & 7,02 & 2,38 & 0 & 100 \\
\hline
\end{tabular}

Fuente: Elaboración propia.

Para identificar el estatus de tratamiento (es decir, si la empresa participó en algún programa público de apoyo a la innovación) la base contiene un total de cuatro variable binarias. Las primeras tres indican la recepción de un beneficio proveniente del Fondo Tecnológico Argentino (FONTAR), el Fondo Fiduciario de Promoción de la Industria del Software (FONSOFT) y la Ley de Promoción de la Industria del Software, respectivamente, mientras que la última recoge la participación en cualquiera de los tres programas anteriores (BENEF). La distribución de frecuencias (véase el cuadro 2) revela que, según las declaraciones de las empresas relativas al año 2010, el 23\% recibió un beneficio del 
FONTAR, el 49\% participó en el FONSOFT y el 35\% estaba inscrita en la Ley de Promoción de la Industria del Software. De manera global, más allá del tipo de instrumento, el 65\% recibió un beneficio para investigación, desarrollo e innovación (l+D+l).

\section{Cuadro 2}

Argentina: participación de las empresas de software y servicios informáticos en programas públicos, 2010

(En porcentajes)

\begin{tabular}{lcccc}
\hline & FONTAR & FONSOFT & $\begin{array}{c}\text { Ley de Promoción de la } \\
\text { Industria del Software }\end{array}$ & BENEF \\
\hline No beneficiarias & 77,25 & 51,32 & 64,55 & 34,39 \\
\hline Beneficiarias & 22,75 & 48,68 & 35,45 & 65,61 \\
\hline Total & 100 & 100 & 100 & 100 \\
\hline
\end{tabular}

Fuente: Elaboración propia.

El reducido tamaño de la muestra impide identificar de manera robusta los posibles efectos diferenciales de los distintos programas de financiamiento en el comportamiento innovador y económico de las empresas de software y servicios informáticos. En consecuencia, solo se estima el efecto medio del tratamiento en los tratados de la participación en cualquiera de los programas de apoyo al sector (variable BENEF).

En relación con las variables de control, la base de datos permite diseñar un conjunto heterogéneo de variables que pueden afectar la posibilidad de recibir apoyo a la innovación. Como variable sustitutiva del tamaño de la empresa se consideró el número de empleados en 2008. Dado que la variable presenta una distribución sesgada se la incorporó tomada en logaritmo (I_size). Para representar la antigüedad de la empresa se construyó una variable que mide la cantidad de años que transcurrieron desde el inicio de sus actividades hasta el año 2008 y se corrigió el sesgo en su distribución empleando una vez más la forma logarítmica (I_age). Para completar la caracterización estructural del sector se incorporaron dos variables: un indicador dicotómico relativo a la pertenencia de la empresa a un grupo empresarial (cap_ext) y otro, también dicotómico, para indicar si la empresa está localizada en Buenos Aires (bsas). Como variable sustitutiva de la capacidad de absorción de la empresa (Cohen y Levinthal, 1990) se creó una variable binaria que indica la presencia de empleados con posgrado (cap_abs). Asimismo, se incorporaron tres variables relacionadas con aspectos de la conducta innovadora de la empresa. La primera es una variable binaria que indica si la empresa realiza esfuerzos de innovación a través de la I+D interna (internal_r\&d), la segunda recoge la cantidad de vínculos establecidos con otras empresas para actividades de I+D (link_firms) y la tercera es la cantidad de vínculos que declaró la empresa con instituciones públicas - como universidades o centros tecnológicos ${ }^{1}$ (link_publ) - . Es de esperar que estas últimas variables incidan positivamente en la probabilidad de recibir un beneficio público. Es decir, por una parte, se espera que las empresas que realizan esfuerzos de innovación tengan una conducta innovadora más activa y proclive a solicitar y obtener financiamiento público y, por otra, que las empresas que integran redes (junto a otras empresas, universidades o centros tecnológicos) para complementar sus capacidades internas sean muy activas en la búsqueda de financiamiento mediante los instrumentos propuestos por el sector público.

Por último, el conjunto de variables de resultado contempla las dimensiones innovadora y económica del desempeño de las empresas. En relación con el primer tipo de desempeño se proponen las siguientes cuatro variables sustitutivas: i) una variable binaria que toma el valor 1 si la empresa introdujo un nuevo producto o servicio en el mercado (inno); ii) la razón entre la inversión de

\footnotetext{
1 Para evitar una correlación espuria no se tuvieron en cuenta los vínculos con el Ministerio de Industria o la Agencia Nacional de Promoción Científica y Tecnológica del Ministerio de Ciencia, Tecnología e Innovación Productiva.
} 
la empresa en actividades de I+D y sus ventas (r\&d_share); iii) la razón entre el número de empleados dedicados a tareas de I+D y el total de empleados (emp_r\&d), y iv) la razón entre las ventas del producto o servicio innovador en dólares y el número de ocupados en el departamento de I+D (r\&d_ product). En relación con el desempeño económico de las empresas se consideran tres aspectos: i) productividad del trabajo: medida como la razón de ventas por ocupado (product); ii) desempeño exportador: evaluado mediante dos indicadores, uno binario que indica si la empresa exporta (expo_ bin) y otro continuo que indica el coeficiente exportador de la empresa (expo_share), y iii) desempeño laboral: basado en la tasa de crecimiento acumulada desde 2008 hasta 2010 (emp_crec).

En el cuadro 3 se presenta la estadística descriptiva de las variables de la muestra. En la gran mayoría de los casos el valor medio de las variables difiere significativamente entre las empresas del grupo de control y las del grupo de tratamiento. Por ejemplo, las empresas que recibieron algún apoyo de los programas analizados se caracterizaron por un comportamiento innovador más proactivo, que se manifestó en mayores índices de actividades internas de I+D, vínculos con otras empresas e instituciones y capacidades de absorción. En relación con el conjunto de variables de resultado, pese a que la prueba de medias arroja diferencias significativas, estas no pueden atribuirse a la participación en el programa público. Las diferencias sistemáticas entre ambos grupos con respecto al grupo de covariables sugieren que las empresas beneficiarias son sustancialmente diferentes de sus pares del grupo de control, de manera que la comparación directa estaría sesgada. La técnica de balanceo aplicada en este artículo permite seleccionar un grupo de control con características observables similares a las de las empresas beneficiarias. Tras este ejercicio, las diferencias entre ambos grupos pueden - bajo ciertos supuestosexplicarse a partir de la participación en el programa público de apoyo.

Cuadro 3

Diferencias de medias entre empresas tratadas y no tratadas

\begin{tabular}{lcc}
\hline & \multicolumn{2}{c}{ BENEF } \\
\cline { 2 - 3 } & Diferencia & Valor $p$ \\
\hline Covariables & & \\
\hline I_size & 0,31 & 0,13 \\
\hline I_age & 0 & 0,83 \\
\hline cap_ext & $-3,24$ & 0,38 \\
\hline Bsas & 0,11 & 0,10 \\
\hline cap_abs & 0,06 & 0,67 \\
\hline internal_r\&d & 0,22 & 0,00 \\
\hline link_firms & 0,12 & 0,35 \\
\hline link_publ & 0,44 & 0,00 \\
\hline Desempeño innovador & & \\
\hline prod_inno & 25,33 & 0,00 \\
\hline r\&d_share & 3,84 & 0,42 \\
\hline emp_r\&d & 0,04 & 0,78 \\
\hline r\&d_produc & $-72,904$ & 0,33 \\
\hline Desempeño económico & & \\
\hline Product & 0,15 & 0,38 \\
\hline expo_bin & 0,17 & 0,03 \\
\hline expo_share & 0,26 & 0,00 \\
\hline emp_crec & 0,01 & 0,09 \\
\hline
\end{tabular}

Fuente: Elaboración propia. 


\section{Marco metodológico}

\section{Un enfoque exploratorio desde la perspectiva de los diagramas causales}

El herramental de grafos acíclicos dirigidos (DAG) permite mostrar las dificultades que enfrenta la inferencia causal cuando se evalúan los efectos de participar en un programa de apoyo a la innovación. En términos formales, un grafo acíclico dirigido (Pearl, 2000) es una representación gráfica de los supuestos cualitativos de las relaciones causales que el investigador tiene en mente. En términos más simples, es una red compuesta por nodos (variables), flechas (propuestas de relaciones causales) y flechas ausentes (propuestas de inexistencia de relaciones causales). A esa red se le puede agregar un conjunto de supuestos y reglas que ayuden a relacionar rigurosamente ese grafo con datos y modelos econométricos.

En el gráfico 1 se muestra el DAG entre i) la participación en un programa público de financiamiento a la I+D; ii) la conducta innovadora, y iii) el desempeño económico de la empresa. En este diagrama, cada nodo representa una variable (o conjunto de variables) con su correspondiente función de distribución. La flecha indica la dirección del efecto causal entre esas dos variables: si la flecha va desde la innovación al desempeño, es porque se propone que este último es afectado causalmente por la primera. La utilidad de este diagrama es que permite ilustrar las dificultades para identificar el efecto causal asociado a la participación en un programa público.

En primer término, se propone una relación causal entre la recepción de financiamiento para actividades de innovación (programa público) y la conducta innovadora de la empresa (innovación). En el diagrama se señala un conjunto de covariables que afectan tanto la participación en el programa público como la introducción de nuevos productos o servicios. Ese grupo de covariables está integrado por factores observables, como el tamaño de la empresa, su antigüedad, el origen del capital, la localización geográfica, la realización de actividades internas de I+D, la presencia de posgraduados y los vínculos con otros actores (empresas, universidades o centros públicos de investigación). Planteado este DAG, el uso de un modelo econométrico para estimar la relación entre el programa y la innovación producirá un resultado sesgado e inconsistente, pues el efecto causal se puede "confundir" con el impacto de las variaciones en el conjunto de covariables observables. En consecuencia, para estimar el efecto causal del programa público se proponen dos estrategias de identificación y estimación: i) una estimación no paramétrica basada en el balanceo de la base de datos según el puntaje de propensión a participar en el programa público, y ii) una estimación paramétrica doblemente robusta (Lunceford y Davidian, 2004).

Gráfico 1

Diagrama acíclico de relaciones causales

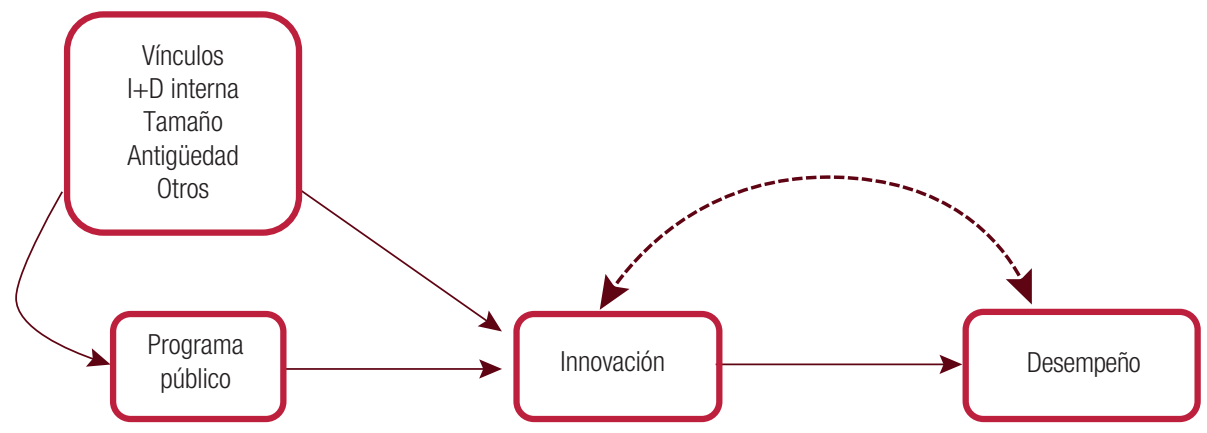

Fuente: Elaboración propia. 
En segundo término, en el diagrama se plantea que la relación entre el programa público y el desempeño económico de la empresa está mediada por su desempeño innovador. En consecuencia, para estimar el impacto debería utilizarse el producto entre el efecto del programa en la innovación, por una parte, y el efecto de la innovación en el desempeño, por otra. Sin embargo, dado que estas últimas dos variables se determinan de manera simultánea por la presencia de factores inobservables comunes (la flecha bidireccional punteada), la estimación directa antes propuesta arrojará resultados sesgados e inconsistentes. Así, para identificar este último efecto se deberá adoptar una estrategia de variables instrumentales en la que el grupo de instrumentos esté formado por el conjunto de covariables y la participación en el programa público.

\section{Estrategia de identificación: emparejamiento por puntaje de propensión}

La literatura sobre evaluación de impacto ofrece un variado menú de técnicas para estimar el efecto causal de un programa público². Entre ellas destacan las siguientes estrategias de estimación paramétricas y no paramétricas: i) diferencias en diferencias; ii) modelos de selección; iii) variables instrumentales; iv) técnicas de emparejamiento basadas en un puntaje de propensión, y v) regresión discontinua.

En este caso, la estructura de la base de datos (presentada y analizada en la próxima sección) restringe este amplio menú. Al no contar con una base de datos de corte transversal y temporal (micropanel), no es posible estimar un modelo de diferencias en diferencias. Asimismo, debido a la falta de un conjunto de instrumentos válidos para instrumentar la participación en el programa de apoyo, tampoco es posible llevar adelante un estudio de inferencia causal basado en métodos de variables instrumentales. Lo mismo sucede con la posibilidad de construir una ecuación de exclusión para estimar un modelo de selección. Por lo tanto, la naturaleza de corte transversal de la base solo permite el empleo de una técnica de emparejamiento basada en un puntaje de propensión (emparejamiento por puntaje de propensión).

Para comenzar, se asume que existe una variable de interés para la empresa $i, Y_{i}$ (decisión de innovar o gasto en I+D, por ejemplo), que puede adoptar dos valores en función de su participación en un programa público de financiamiento. Luego, se define $D_{i}$ como una variable binaria que toma el valor 1 si la empresa $i$ participó en el programa y 0 si no lo hizo. De este modo, la variable de interés puede definirse de la siguiente manera:

$$
Y_{i}=\left\{\begin{array}{l}
Y_{i 0} \text { si } D_{i}=0 \\
Y_{i 1} \text { si } D_{i}=1
\end{array}\right.
$$

Asimismo, el efecto causal medio de la participación en el programa de financiamiento se puede definir de la siguiente forma:

$$
E\left(\alpha_{T T}\right)=E\left[\left(Y_{i 1}-Y_{i 0}\right) / D_{i}=1\right]=E\left(Y_{i 1} / D_{i}=1\right)-E\left(Y_{i 0} / D_{i}=1\right)
$$

Cuando se evalúa el efecto causal de una política las preguntas a las que se busca responder son: ¿qué hubiera pasado con los beneficiarios si el programa no hubiera existido?, ¿qué hubiera pasado con el gasto en actividades de I+D si el programa examinado no se hubiera adoptado? Desde una perspectiva econométrica esto plantea un problema de datos faltantes. Si bien podemos observar directamente $E\left(Y_{i 1} / D_{i}=1\right)$ (el valor medio de la variable objetivo entre las empresas que

2 Véanse Heckman, Lalonde y Smith (1999), Imbens y Wooldridge (2009) y un abordaje más amplio en Morgan y Winship (2007). 
participaron del programa), no sucede lo mismo con $E\left(Y_{i 0} / D_{i}=1\right)$ (el valor que hubiera registrado la variable objetivo si la misma empresa $i$ no hubiera participado en el programa). Solo se puede ver lo que ocurrió y no lo que hubiera ocurrido con la variable objetivo sin el programa. Esa situación hipotética recibe el nombre de contrafactual, que al no poder observarse debe estimarse empleando el subgrupo de empresas que no participó en el programa: $\widehat{Y_{i 0}}$. Así, la fórmula para calcular el efecto medio del tratamiento en las empresas participantes del programa se modifica de la siguiente forma:

$$
E\left(\alpha_{T T}\right)=E\left[\left(Y_{i 1}-\widehat{Y_{i 0}}\right) / D_{i}=1\right]
$$

Como los beneficiarios del programa no fueron asignados aleatoriamente, no es posible descartar la posibilidad de un sesgo de selección al estimar el impacto de $D_{i}$ sobre $Y_{i}$. En consecuencia, no se puede afirmar $E\left(Y_{i 0} / D_{i}=1\right) \neq E\left(Y_{i 0} / D_{i}=0\right)$ ni estimar el contrafactual utilizando el promedio simple de las empresas que no recibieron subsidios o beneficios fiscales. Para solucionar este problema se puede recurrir al supuesto de independencia condicional (Rubin, 1977), según el cual tanto la participación en un programa público como su potencial resultado son estadísticamente independientes para empresas con un mismo conjunto de características observables, $X$. Así $E\left(Y_{i 0} / D_{i}=1, X\right)=E\left(Y_{i 0} / D_{i}=0, X\right)$ y las diferencias entre ambos grupos solo son atribuibles al programa. Hechas estas precisiones, el efecto medio del tratamiento puede estimarse como:

$$
E\left(\alpha_{T T}\right)=E\left(Y_{i 1} / D_{i}=1, X=x\right)-E\left(Y_{i 0} / D_{i}=0, X=x\right)
$$

Cabe mencionar algunos puntos importantes. Además del supuesto de independencia condicional, otro prerrequisito para la consistencia del emparejamiento es que haya suficiente grado de solapamiento entre el grupo de control y el grupo de empresas que recibieron el subsidio. Es necesario que el grupo de control contenga al menos una unidad suficientemente similar a cada empresa tratada. En la práctica, esta condición se logra restringiendo la muestra a un soporte común. Para ello se calculan los umbrales mínimo y máximo del puntaje de propensión y se eliminan las observaciones cuyo puntaje esté fuera de esos límites inferior y superior.

El ejercicio de emparejar cada empresa que participó en el programa introduce un problema adicional conocido como "la maldición de la dimensionalidad". La lista de factores observables que inciden tanto en la participación en el programa como en el resultado estudiado puede ser demasiado extensa y hacer casi imposible el emparejamiento para cada unidad individual de manera separada. A medida que crece el conjunto de factores observables usados durante el procedimiento de emparejamiento, la probabilidad de encontrar un control exacto disminuye de manera exponencial. A su vez, es muy fácil probar en la práctica que la aplicación del método de emparejamiento mediante un conjunto relativamente pequeño de factores produce un conjunto de beneficiarios para el cual no existe un emparejamiento posible. Rosenbaum y Rubin (1983) sugirieron realizar el procedimiento de emparejamiento entre beneficiarios y no beneficiarios usando únicamente su puntaje de propensión (la estimación de la probabilidad condicional de participar en el programa). Esto reduce el procedimiento de emparejamiento concebido como un problema multidimensional (en el que la dimensión depende del número de variables del problema) a un problema unidimensional. En la práctica esto se realiza mediante una estimación de máxima verosimilitud para la probabilidad de participar en el programa en función de un conjunto de covariables.

Sin embargo, calcular el puntaje de propensión correspondiente a cada empresa de la muestra no basta. Emparejar dos empresas con un puntaje idéntico es casi imposible, dado que se trata de una variable continua. En ese sentido, la literatura econométrica documenta el desarrollo de diversos métodos para solucionar este problema. En este artículo se utiliza el método de emparejamiento de Kernel: cada empresa que participó en el programa se empareja con un promedio ponderado de 
todas las empresas del grupo de control, donde el peso que reciben es inversamente proporcional a la distancia entre el puntaje de propensión de la empresa tratada y la empresa del grupo control.

En resumen, la idea básica de la técnica de emparejamiento por puntaje de propensión consiste en la construcción de grupos de control mediante procedimientos estadísticos de emparejamiento (matching). Esta metodología corrige las diferencias observables entre el grupo de tratamiento (beneficiarios del programa) y el grupo de control (no beneficiarios del programa) buscando para cada unidad individual de la muestra del grupo de tratamiento la unidad individual más parecida de la muestra de no beneficiarios, que conformarán el grupo de control. De esta manera, las diferencias entre ambos grupos solo pueden atribuirse a la participación en el programa público. El principal supuesto de esta metodología es que la participación se basa en características observables de las unidades individuales. De no ser así, los resultados de la evaluación obtenidos con esta metodología estarán sesgados. La fuente de sesgo reside en la potencial correlación entre las variables no observables, que afectan tanto la decisión de participación del individuo en el programa como la variable de interés de la evaluación. En el cuadro 4 se presenta un resumen esquemático de las etapas involucradas en este método.

Cuadro 4

Pasos del emparejamiento por puntaje de propensión

\begin{tabular}{ll}
\hline & Especificar y estimar un modelo probit para la participación en el programa. Generar \\
Paso 1 & la probabilidad predicha para cada unidad de la muestra.
\end{tabular}

Paso 2 Restringir la muestra a un soporte común. Se eliminan todas las empresas que recibieron algún subsidio o beneficio

Paso 2 fiscal y tienen un puntaje superior o inferior al puntaje máximo o mínimo, respectivamente, del grupo control.

Estimar el efecto medio del tratamiento en los tratados utilizando el método de Kernel a partir de la siguiente fórmula:

$$
\alpha_{T T}^{\text {kernel }}=\frac{1}{N^{T}} \sum_{i \in T}\left\{Y_{i}^{T}-\frac{\sum_{j \in C} Y_{j}^{C} G\left(\frac{p_{j}-p_{i}}{h_{n}}\right)}{\sum_{k \in C} G\left(\frac{p_{k}-p_{i}}{h_{n}}\right)}\right\}
$$

Paso 3

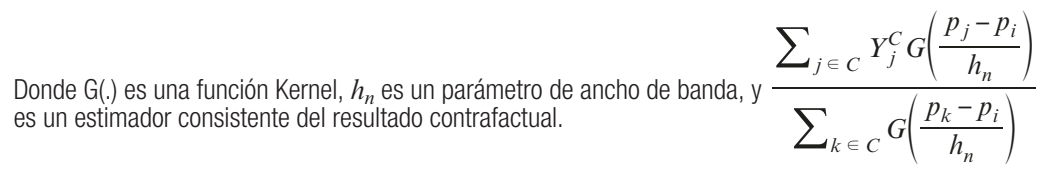

Paso 4 Se aplica la técnica de bootstrap para calcular el error estándar asociado a cada efecto medio del tratamiento en los tratados y se verifica si la diferencia de medias es estadísticamente distinta de cero.

Fuente: Elaboración propia, sobre la base de D. Czarnitzki y C. Lopes-Bento, "Value for money? New microeconometric evidence on public R\&D grants in Flanders", Research Policy, vol. 42, № 1, Amsterdam, Elsevier, 2013.

\section{Estrategia de identificación: estimación doblemente robusta}

Robins y otros colegas (Bang y Robins, 2005; Robins, Rotnitzky y Zhao, 1995; Robins, 2000) introdujeron el concepto de estimaciones doblemente robustas. Esta técnica requiere que tanto el modelo para estimar el puntaje de propensión de cada empresa como el modelo para estimar la variable resultado sean utilizados en un mismo estimador. Los estimadores calculados de esta manera reciben el nombre de estimadores eficientes semiparamétricos. Tsiatis (2006) y Leon, Tsiatis y Davidian (2003) muestran que son estimadores doblemente robustos porque proporcionan estimaciones del efecto medio del tratamiento en los tratados consistentes siempre que se cumpla por lo menos una de las siguientes condiciones: i) que el modelo del puntaje de propensión esté correctamente especificado (es decir, que $\hat{p}_{i}$ sea el verdadero puntaje de propensión), y ii) que el modelo de regresión 
que relaciona la variable resultado con las covariables esté correctamente especificado. En el caso de que ambos modelos estén bien especificados, las estimaciones del estimador semiparamétrico tendrán la menor varianza. Lunceford y Davidian (2004) proponen la siguiente fórmula para calcular estimaciones doblemente robustas:

$$
\hat{\tau}_{D R}=\frac{1}{N} \sum_{i=1}^{N} \frac{A_{i} Y_{i}-\left(A_{i}-\hat{p}_{i}\right) m_{1}\left(\underline{X}_{i}\right)}{\hat{p}_{i}}-\frac{1}{N} \sum_{i=1}^{N} \frac{\left(1-A_{i}\right) Y_{i}+\left(A_{i}-\hat{p}_{i}\right) m_{0}\left(\underline{X}_{i}\right)}{1-\hat{p}_{i}}
$$

Donde $\hat{p}_{i}$ es una estimación de la probabilidad de participar en el programa público, $m_{A}=\left(\underline{X}_{i}\right)=E\left(Y_{i} \mid A_{i}=A, \underline{X}_{i}\right)$ para $A=0 \circ A=1$ son los valores predichos provenientes de regresiones separadas de la variable resultado como función del conjunto de covariables.

\section{Evaluación del efecto causal de la participación en un programa}

\section{Efecto en la conducta innovadora: estimación del efecto medio del tratamiento en los tratados mediante el emparejamiento por puntaje de propensión}

Como se resaltó en la sección metodológica, el primer paso para calcular el efecto medio del tratamiento en los tratados consiste en balancear la muestra entre empresas beneficiarias y no beneficiarias sobre la base de los factores observables. Para ello es necesario estimar la probabilidad de que una empresa de software y servicios informáticos participe en alguno de los programas públicos analizados en función de sus características observables.

Para lograr el emparejamiento entre empresas beneficiarias y no beneficiarias se estimó la probabilidad de participar en algún programa público utilizando un modelo probit. En el cuadro 5 se presenta el efecto marginal de cada una de las variables propuestas en la probabilidad predicha. La prueba de significatividad conjunta valida en todos los casos el modelo presentado y sugiere un conjunto de resultados bastante heterogéneo, que varía según el programa público analizado. Por ejemplo, la participación en el FONTAR está afectada positivamente por el tamaño de la empresa en 2008, las actividades de I+D interna y los vínculos que mantuvo tanto con otras empresas como con instituciones públicas, entre ellas universidades y centros tecnológicos. Con respecto al FONSOFT, los resultados indican que la probabilidad de recibir el beneficio muestra diferencias significativas en función de la localización geográfica y crece en función de los vínculos con universidades y centros tecnológicos y la realización de actividades de I+D interna. A su vez, la probabilidad de recibir los beneficios fiscales que otorga la Ley de Promoción de la Industria del Software es afectada positivamente por el tamaño y aumenta en virtud de los vínculos con otras empresas para hacer I+D. Asimismo, la probabilidad es sistemáticamente mayor para las empresas que realizan actividades de I+D interna y - paradójicamente - menor para aquellas que tienen personal con posgrado. El último modelo analiza la probabilidad de participar en cualquiera de estos programas. Se observan diferencias significativas en función de la localización geográfica de la empresa y un impacto positivo de las actividades de I+D interna y los vínculos con otras empresas o instituciones públicas para hacer I+D. 
Cuadro 5

Efectos marginales del modelo probit en la variable ficticia de participación

\begin{tabular}{lc}
\hline & BENEF \\
\hline I_size & 0,015 \\
\hline I_age & 2676 \\
\hline cap_ext & $-0,001$ \\
\hline Bsas & $0,155^{\star}$ \\
\hline cap_abs & $-0,025$ \\
\hline internal_r\&d & $0,215^{\star \star}$ \\
\hline link_firms & $0,012^{\star \star}$ \\
\hline link_publ & $0,426^{\star \star *}$ \\
\hline Wald chi2(8) & 43295 \\
\hline Prob $>$ chi2 & 0,0000 \\
\hline$N$ & 187 \\
\hline
\end{tabular}

Fuente: Elaboración propia.

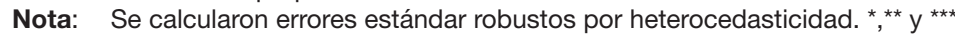
indican niveles de significatividad al $10 \%$, el $5 \%$ y el $1 \%$, respectivamente.

Como se destacó anteriormente, un prerrequisito importante es que para cada empresa receptora de un beneficio exista una observación de control con un puntaje de propensión bastante similar (restricción de soporte común). Para ello se calcularon los valores mínimo y máximo del puntaje de propensión para las empresas del grupo de control, lo que permitió eliminar cuatro empresas del grupo de tratamiento que no contaban con empresas similares en el grupo de control ${ }^{3}$. En consecuencia, esas empresas no se tuvieron en cuenta en el proceso de emparejamiento. Dado que las observaciones perdidas no constituyen una parte sustancial de la muestra, la restricción de soporte común no afecta los resultados de manera significativa. Además de la imposición de un soporte común, el supuesto de independencia condicional sostiene que, condicionadas por el puntaje de propensión, las diferencias entre empresas beneficiarias y no beneficiarias solo son atribuibles al programa público objeto de estudio. Desde otro ángulo, este supuesto implica que las diferencias entre ambos grupos de empresas basadas en factores observables desaparecen. Así, se exige que las covariables propuestas para explicar la participación en el programa público estén balanceadas entre ambos grupos. En el cuadro 6 se presenta el valor $p$ asociado a la prueba de medias y se sugiere que no puede rechazarse la hipótesis nula sobre la inexistencia de diferencias entre empresas beneficiarias y no beneficiarias.

A pesar de estos resultados, considerar que la calidad del emparejamiento es buena solo a partir de la prueba de medias es muy desaconsejable 4 . En ese sentido, de acuerdo con Rosenbaum y Rubin (1983), se procedió a la estimación del sesgo medio estandarizado después del emparejamiento para evaluar su calidad. El sesgo estandarizado se define como la diferencia de medias en las dos situaciones (tratamiento y no tratamiento), dividida por la raíz cuadrada de la media de las varianzas respectivas. El consenso ha establecido un límite del 10\% para evaluar la calidad del emparejamiento. En el cuadro 7 se presenta el porcentaje de sesgo para cada covariable. En líneas generales este criterio se cumple, con excepción de algunos casos puntuales.

3 Esta afirmación solo es válida para el FONTAR, pues en el resto de los programas analizados no hubo unidades fuera de la región de soporte común.

4 Cabe recordar que la prueba de medias se realiza sobre supuestos muy fuertes, como la distribución normal de las variables (que claramente no se cumple cuando hay variables binarias o multinomiales), y es muy sensible a la cantidad de datos que tenga la muestra. 
Cuadro 6

Calidad del emparejamiento: valor $p$ de la prueba de medias

\begin{tabular}{ll}
\hline & BENEF \\
\hline I_size & 0,686 \\
\hline I_age & 0,78 \\
\hline cap_ext & 0,818 \\
\hline Bsas & 0,775 \\
\hline cap_abs & 0,228 \\
\hline linternal_r\&d & 0,712 \\
\hline link_publ & 0,646 \\
\hline
\end{tabular}

Fuente: Elaboración propia.

Cuadro 7

Calidad del emparejamiento: porcentaje del sesgo estandarizado

\begin{tabular}{lc}
\hline & BENEF \\
\hline I_size & $-5,1$ \\
\hline I_age & 3,7 \\
\hline cap_ext & 2,3 \\
\hline Bsas & $-3,7$ \\
\hline cap_abs & 7,7 \\
\hline internal_r\&d & $-4,2$ \\
\hline link_firms & $-6,2$ \\
\hline link_publ & 3,2 \\
\hline
\end{tabular}

Fuente: Elaboración propia.

A modo de conclusión, luego de evaluar los resultados de la prueba de medias junto con los valores del sesgo estandarizado puede afirmarse que todas las covariables propuestas están bien balanceadas después del emparejamiento. Se concluye que el emparejamiento fue exitoso y se procede a estimar el efecto medio del tratamiento en los tratados. De existir diferencias estadísticamente significativas, estas podrán atribuirse al beneficio recibido.

Podría argumentarse que los resultados anteriores carecen de robustez debido a la baja cantidad de empresas beneficiarias que contiene la muestra en algunos instrumentos. En ese sentido, se propone una cuarta estimación, en la que el efecto medio del tratamiento en los tratados se calcula teniendo en cuenta la participación en cualquiera de los programas públicos anteriores. Los cálculos muestran que para las empresas del sector de software y servicios informáticos, la recepción de algún beneficio implicó un incremento medio del gasto en actividades de I+D (medido como porcentaje de las ventas) de casi 6 puntos porcentuales con respecto al escenario que se hubiera obtenido en ausencia de la ayuda estatal. El peso del personal del equipo de I+D en la plantilla de la empresa también registró un salto positivo gracias a la participación en los programas públicos de apoyo: la estimación muestra un incremento de 11 puntos porcentuales. Por último, la estimación del efecto medio del tratamiento en los tratados reveló que la probabilidad de introducir un nuevo producto en el mercado aumentó en promedio un $20 \%$ con respecto al valor registrado en ausencia de beneficios (véase el cuadro 8). 
Cuadro 8

Estimación del efecto medio del tratamiento en los tratados para programas (global)

\begin{tabular}{lccc}
\hline & N (beneficiarios) & Efecto (sin emparejar) & Efecto (emparejado) \\
\hline prod_inno & 124 & 25,00 & $19,00^{* *}$ \\
\hline r\&d_share & 124 & 5,28 & $5,45^{*}$ \\
\hline r\&d_produc & 124 & $-72,904$ & $-48,601$ \\
\hline emp_r\&d & 124 & 0,04 & $0,11^{* *}$ \\
\hline
\end{tabular}

Fuente: Elaboración propia.

Nota: Se calcularon errores estándar por método de bootstrapping con 300 repeticiones.

${ }^{*},{ }^{* *}{ }^{* \star *}$ indican significatividad estadística al $10 \%$, el $5 \%$ y el $1 \%$, respectivamente.

Vistos en conjunto, los resultados permiten afirmar que los instrumentos públicos de apoyo al sector ayudaron a configurar un grupo de empresas con una conducta innovadora más dinámica. En particular, el mayor gasto en I+D se canalizó a través de la contratación de empleados focalizados en esas actividades. Este conjunto de actividades explica por qué la probabilidad de innovar es sistemáticamente mayor para las empresas que recibieron algún beneficio público. Sin embargo, pese a estos ponderables resultados, no se observaron diferencias significativas en la productividad del equipo de I+D (medida como ventas del producto innovador por empleado del equipo de I+D) tras la recepción del subsidio. Este resultado podría obedecer al breve lapso de tiempo analizado (2008 a 2010), necesitándose más tiempo para que el comportamiento innovador más dinámico se traduzca en mejoras en la productividad del área de I+D.

\section{Efecto en la conducta innovadora: estimación del efecto medio del tratamiento en los tratados mediante estimaciones doblemente robustas}

En esta sección se aplica una técnica alternativa para estimar el efecto medio de participar en un programa público de apoyo a la I+D, conocida como estimaciones doblemente robustas.

En primer lugar, se introducen dos modificaciones al grupo de variables consideradas. Por una parte, la variable de tratamiento toma el valor 1 si la empresa participa en cualquiera de los programas de financiamiento estudiados (FONTAR, FONSOFT o Ley de Promoción de la Industria del Software). Por otra, el grupo de variables de resultados considerado se restringe a: i) gasto en actividades de I+D como porcentaje de la ventas; ii) personal dedicado a tareas de I+D como porcentaje del total, y iii) introducción de un nuevo producto en el mercado.

La estimación doblemente robusta es otra técnica paramétrica que combina el emparejamiento realizado a partir del puntaje de propensión con un modelo econométrico. El modelo propone una función para cada variable independiente, que incluye las covariables que afectan la decisión de participar en el programa de financiamiento correspondiente y una variable binaria para la participación en algún programa público. Asimismo, se restringe la estimación a las observaciones que pertenecen a la región de soporte común y se pondera cada empresa según su puntaje de propensión. Los resultados presentados en el cuadro 9 permiten afirmar la significatividad global del modelo propuesto (valor $p$ del $0 \%$ ), la significatividad individual de los subsidios o beneficios fiscales y la no significatividad del resto de las variables. Este conjunto de resultados muestra que la estimación del efecto medio del tratamiento en los tratados no está sesgada y es consistente, ya que se controla el sesgo por selección basado en características observables. Se puede apreciar que las estimaciones obtenidas son similares a los resultados alcanzados por métodos no paramétricos que se detallan en el cuadro 8. 
Cuadro 9

Estimación doblemente robusta del efecto medio del tratamiento en los tratados

\begin{tabular}{lccc}
\hline & Gasto en I+D & Recursos humanos en I+D & Innovación de producto \\
\hline Beneficio & $6,003^{*}$ & $0,093^{\star *}$ & $0,212^{\star *}$ \\
\hline Tamaño & -4552 & $-0,167$ & $-0,055$ \\
\hline Antigüedad & 483,09 & $-5,02$ & 6399 \\
\hline I+D interna & $-3,220$ & 0,145 & 0,273 \\
\hline Vínculos para I+D & -2498 & $-0,038$ & 0,013 \\
\hline Vínculos con universidades y otros & -4428 & $-0,008$ & 0,019 \\
\hline Posgraduados & 6549 & $-0,006$ & 0,034 \\
\hline Grupo & 0,003 & 0 & 0,001 \\
\hline Localización geográfica & -2236 & $-0,058$ & $-0,089$ \\
\hline Intercepto & -3639123 & 39097 & -48149 \\
\hline Prob $>$ chi2 & $2,21 \mathrm{E}-07$ & 0,0101238 & 0,0130124 \\
\hline $\mathrm{N}$ & 43 & 173 & 184 \\
\hline
\end{tabular}

Fuente: Elaboración propia.

Nota: Se calcularon errores estándar robustos.

${ }^{*, * *} y^{* \star *}$ indican significatividad estadística al $10 \%$, el $5 \%$ y el $1 \%$, respectivamente.

\section{Efecto en el desempeño económico de la empresa: variables instrumentales}

El principal objetivo de este artículo es determinar el papel de los instrumentos públicos de financiamiento a la I+D en el desempeño reciente del sector de software y servicios informáticos. El enfoque metodológico adoptado proponía que el efecto en el desempeño de la empresa está mediado por la generación de novedad (véase el gráfico 1). Allí se explica que la simultaneidad entre la introducción de un nuevo producto en el mercado y el desempeño económico de la empresa impedía captar ese efecto causal. A partir de eso se proponía utilizar una técnica de variables instrumentales, consistente en generar una variación exógena localizada a partir de variables que son redundantes para explicar el desempeño de la empresa, pero están muy correlacionadas con la probabilidad de innovar.

Para estimar el desempeño de las empresas se tuvieron en cuenta tres dimensiones: i) productividad del trabajo (medida como ventas por ocupado); ii) desempeño exportador (se utilizan dos indicadores: uno binario, que indica si la empresa exporta, y otro continuo, que indica el coeficiente exportador de la empresa), y iii) desempeño laboral (se utilizan dos variables: el número total de ocupados en 2010 y la tasa de crecimiento acumulada desde 2008). El conjunto de factores observables y la participación en el programa público se utilizan para instrumentar el efecto de la innovación.

En el cuadro 10 se presentan los resultados de la segunda etapa del método de variables instrumentales. La proyección de máxima verosimilitud obtenida en la etapa anterior (véase el cuadro 10) se utilizó como instrumento de la innovación. La inferencia estadística confirma que la innovación, y por ende la participación en el programa público, afectaron positivamente el desempeño de la empresa. En particular, los resultados muestran un efecto causal en el desempeño exportador (tanto con respecto a la probabilidad de vender a los mercados internacionales como al coeficiente exportador) y en la tasa de variación del empleo. Por último, no se capta un efecto causal en la productividad laboral. 
Cuadro 10

Efecto de la innovación en el desempeño de las empresas

\begin{tabular}{|c|c|c|c|c|}
\hline & Productividad del trabajo & $\begin{array}{l}\text { Exportación } \\
\text { (binaria) }\end{array}$ & $\begin{array}{l}\text { Coeficiente de } \\
\text { exportación }\end{array}$ & Crecimiento del empleo \\
\hline inno_prod & 0,288 & $2,295^{\star \star \star}$ & $1,770^{\star *}$ & $1,461^{*}$ \\
\hline I_size & $0,172^{\star \star}$ & $0,244^{* \star *}$ & $0,170^{\star *}$ & $-0,139^{*}$ \\
\hline I_age & -17296 & 9727 & 25563 & $69,928^{\star \star \star}$ \\
\hline cap_ext & $-0,010^{\star *}$ & $0,010^{*}$ & $0,013^{\star \star *}$ & $0,017^{\star \star \star}$ \\
\hline bsas & 0,195 & $-0,137$ & $-0,16$ & 0,051 \\
\hline Intercept & & -75965 & & \\
\hline chi2 & 12227 & 20228 & 27154 & 39186 \\
\hline$P$ & 0,0318 & 0,0011 & 0,0001 & 0,0000 \\
\hline N & 171 & 188 & 185 & 182 \\
\hline
\end{tabular}

Fuente: Elaboración propia.

Nota: Se calcularon errores estándar robustos.

${ }^{* \star * *} y^{* \star \star}$ indican significatividad estadística al $10 \%$, el $5 \%$ y el $1 \%$, respectivamente.

\section{Conclusiones}

En los últimos años, el sector de software y servicios informáticos argentino mostró un destacado desempeño que lo convirtió en uno de los sectores más dinámicos del país. Las cifras de crecimiento en las ventas internas, las exportaciones y el empleo fueron muy superiores a las registradas en la industria manufacturera y el resto de los servicios. Hace 15 años era impensable que el sector de software y servicios informáticos emplearía en 2014 más asalariados que el complejo automotor considerado en forma agregada. El presente documento se posiciona en ese contexto y brinda una caracterización general del sector, con el objetivo de entender los factores que determinaron el desempeño innovador y económico de las empresas. En términos generales, ese crecimiento se explica por un conjunto variado de factores asociados al contexto económico local y global de los últimos 15 años. Entre ellos, la política pública de apoyo al sector, implementada por fondos nacionales como FONTAR y FONSOFT y complementada por la sanción de la Ley de Promoción de la Industria del Software de 2004, parece haber desempeñado un papel importante.

De la estadística descriptiva presentada en este documento surge que más de la mitad de las empresas encuestadas tuvieron acceso a algún tipo de beneficio público. Al comparar los grupos de empresas beneficiarias y no beneficiarias se observa que las primeras tienen una razón mayor entre la inversión en I+D y las ventas, cuentan con más certificaciones de calidad y se vinculan con mayor frecuencia con otras empresas e instituciones con el objetivo de acceder a conocimientos externos para complementar sus capacidades internas. En ese sentido, la evidencia hallada apoya la hipótesis de que los programas públicos de estímulo a la innovación ayudaron a configurar un grupo de empresas caracterizadas por una conducta innovadora más dinámica.

Luego se realizó una serie de ejercicios econométricos para evaluar específicamente el papel de los programas públicos en el desempeño innovador y económico de las empresas. Los resultados permiten identificar una cadena de relaciones causales que muestran, en primera instancia, un efecto positivo de los programas de apoyo en la conducta innovadora de las empresas. En algunas dimensiones, como la intensidad del gasto en I+D (ya sea medido como la razón entre el gasto y las ventas o el porcentaje de trabajadores dedicados exclusivamente a tareas de I+D), se registró un importante incremento tras la participación en el programa público en comparación con los niveles que se hubieran alcanzado en ausencia de intervención estatal. Paralelamente, la introducción de un nuevo producto o servicio en el mercado también aumentó gracias a la recepción de fondos para complementar la inversión en actividades de I+D. 
Seguidamente se comprobó que, mediados por el efecto en la probabilidad de innovar, los programas públicos de apoyo al sector también fueron un factor determinante del desempeño de las empresas en el mercado. Cabe precisar que este efecto causal resultó ser significativo en el desempeño de las empresas en los mercados internacionales y en términos del empleo, pero no en su desempeño productivo.

En consecuencia, puede afirmarse que los instrumentos de promoción vertical focalizados en el sector de software y servicios informáticos desempeñaron un papel relevante para explicar su despegue tras la salida de la convertibilidad. La intervención pública y, en particular, la política industrial y tecnológica son factores determinantes que suelen estar subestimados en la literatura en comparación con factores exógenos, como las ganancias de competitividad derivadas de la devaluación, o factores culturales favorables, como el dominio del idioma inglés o el huso horario. En este documento se brinda información cuantitativa que se espera contribuya a revalorizar el papel de la intervención pública, en particular cuando se realiza a partir de instrumentos de diseño vertical como los analizados en este artículo.

En términos metodológicos, es importante señalar dos limitaciones previas al estudio de impacto, que deben tenerse en cuenta al momento de evaluar los resultados. En primer lugar, la muestra no se construyó con el propósito explícito de realizar una evaluación de impacto, sino con el objetivo de analizar en qué medida las capacidades (tecnológicas, organizacionales y de absorción) y los vínculos (con otras empresas, universidades e instituciones públicas de apoyo al sector) de las empresas de software y servicios informáticos explicaban el desempeño innovador y económico de los últimos años. En consecuencia, las variables utilizadas para captar el estatus de tratamiento (por ejemplo, si la empresa participó o no en alguno de los programas analizados) no captaron dicho fenómeno en toda su dimensión. Por ejemplo, no es posible precisar el año en que las empresas accedieron a dichos fondos (solo se sabe que entre 2008 y 2010 participaron en los programas) ni con qué tipo de instrumento lo hicieron (Aportes No Reembolsables (ANR), crédito fiscal o crédito subsidiado). En segundo lugar, la naturaleza de corte transversal de la base de datos impidió el empleo de técnicas de evaluación que arrojen resultados más precisos, como por ejemplo un estudio de diferencias en diferencias. Sin embargo, conscientes de estas limitaciones, al diseñar la evaluación de impacto se propuso emplear todas las técnicas pasibles de ser utilizadas: regresión con controles, emparejamiento por puntaje de propensión y estimaciones doblemente robustas. Los resultados estimados fueron bastante similares entre las distintas técnicas, lo que arroja evidencia en favor de su robustez. A su vez, las limitaciones señaladas motivan una recomendación muy importante sobre la disponibilidad de bases de datos para llevar a cabo ejercicios de evaluación de políticas. Esto es, se necesitan bases de datos diseñadas de manera que midan las variables de resultado en distintos momentos del tiempo y puedan cruzarse con la información del padrón de empresas beneficiarias (para una determinación más precisa de los grupos de tratamiento y control).

Por último, este documento plantea un conjunto de interrogantes que marcan la agenda de investigación futura sobre el papel de los instrumentos de apoyo en el sector de software y servicios informáticos. En primer lugar, es importante profundizar el análisis de las empresas que acceden a más de un programa de apoyo a la innovación. Los resultados presentados indican que la simultaneidad de programas no afecta el desempeño innovador de las empresas. Esto plantea la necesidad de una mayor complementación entre los organismos ejecutores de la política pública para aumentar la eficiencia y evitar la superposición de incentivos. Visto que es esperable que las empresas que solicitan y reciben más de un beneficio al mismo tiempo sean las de mejor desempeño, se debería evaluar en qué medida no se requieren estímulos diferenciados según el crecimiento de las empresas. En segundo lugar, después de 15 años de crecimiento continuo del sector de software y servicios informáticos, sería importante promover un debate sobre su orientación estratégica futura. Esto es, definir una especialización hacia los segmentos que han tenido más éxito (financiero, seguridad y macrodatos, entre otros) y fomentar la creación de una masa crítica de empresas que definan un perfil de especialización más complejo. Los instrumentos públicos deberían rediseñarse sobre la base de dicho debate con el objetivo de focalizar el apoyo en esos segmentos estratégicos. 


\section{Bibliografía}

Bang, H. y J.M. Robins (2005), "Doubly robust estimation in missing data and causal inference models", Biometrics, vol. 61, № 4, Washington, D.C., International Biometric Society.

Barletta, F. y otros (2013), "Argentina: dinámica reciente del sector de software y servicios informáticos", Revista CEPAL, № 110 (LC/G.2572-P), Santiago, Comisión Económica para América Latina y el Caribe (CEPAL).

Borello, J. y otros (2005), "Competencias técnicas de los trabajadores informáticos", Revista de la CEPAL, № 87 (LC/G.2287-P), Santiago, Comisión Económica para América Latina y el Caribe (CEPAL).

Castro, L. y D. Jorrat (2013), "Evaluación de impacto de programas públicos de financiamiento sobre la innovación y la productividad. El caso de los servicios de software e informáticos de la Argentina", Documento de Trabajo, № 115, Centro de Implementación de Políticas Públicas para la Equidad y el Crecimiento (CIPPEC).

Chudnovsky, D., A. López y S. Melitsko (2001), "El sector de software y servicios informáticos (SSI) en la Argentina: situación actual y perspectivas de desarrollo", Documento de Trabajo, Nㅜ 27, Buenos Aires, Centro de Investigaciones para la Transformación (CENIT).

Cohen, W.M. y D.A. Levinthal (1990), "Absorptive capacity: a new perspective on learning and innovation", Administrative Science Quarterly, vol. 35, N 1, SAGE Publications.

Czarnitzki, D. y C. Lopes-Bento (2013), "Value for money? New microeconometric evidence on public R\&D grants in Flanders", Research Policy, vol. 42, № 1, Amsterdam, Elsevier.

Heckman, J., R. Lalonde y J. Smith (1999), "The economics and econometrics of active labor market programs", Handbook of Labor Economics, vol. 3, O. Ashenfelter y D. Card (eds.), Amsterdam, Elsevier.

Imbens, G.W. y J.M. Wooldridge (2009), "Recent developments in the econometrics of program evaluation", Journal of Economic Literature, vol. 47, № 1, Nashville, Tennessee, American Economic Association.

Leon, S., A.A. Tsiatis y M. Davidian (2003), "Semiparametric estimation of treatment effect in a pretest-posttest study", Biometrics, vol. 59, № 4, Washington, International Biometric Society.

López, A. (2003), "Innovación y propiedad intelectual en la industria del software y los servicios informáticos. Situación y perspectivas para los países en desarrollo", documento presentado en la Reunión Regional OMPI-CEPAL sobre el Sistema Nacional de Innovación: Propiedad Intelectual, Universidad y Empresa, Santiago.

López, A. y D. Ramos (2008), La industria de software y servicios informáticos argentina. Tendencias, factores de competitividad y clusters, Buenos Aires, Centro de Investigaciones para la Transformación (CENIT).

Lunceford, J.K. y M. Davidian (2004), "Stratification and weighting via the propensity score in estimation of causal treatment effects: a comparative study", Statistics in Medicine, vol. 23, No 19, Wiley.

Maldonado, M.U., H.A. Morero y C. Borrastero (2013), "'Catching up' en servicios intensivos en conocimiento: el caso de la producción de software y servicios informáticos de Argentina y Brasil”, Revista Iberoamericana de Ciencia, Tecnología y Sociedad, № 8(24), Buenos Aires, Centro de Estudios sobre Ciencia, Desarrollo y Educación Superior.

Morgan, S. y C. Winship (2007), Counterfactuals and Causal Inference: Methods and Principles for Social Research, Cambridge, Cambridge University Press.

Pearl, J. (2000), Causality: Models, Reasoning, and Inference, Cambridge, Cambridge University Press.

Perazzo, R. y otros (1999), "Oportunidades para la producción y exportación argentina de software", Documento de Trabajo, № 9, Buenos Aires, Agencia Nacional de Promoción Científica y Tecnológica.

Robins, J.M. (2000), "Robust estimation in sequentially ignorable missing data and causal inference models", Proceedings of the American Statistical Association Section on Bayesian Statistical Science 1999, Alexandria, American Statistical Association.

Robins, J.M., A. Rotnitzky y L.P. Zhao (1995), "Analysis of semiparametric regression-models for repeated outcomes in the presence of missing data", Journal of the American Statistical Association, vol. 90, № 429, Taylor \& Francis.

Rosenbaum, P.R. y D.B. Rubin (1983), "The central role of the propensity score observational studies for causal effects", Biometrika, vol. 70, № 1.

Rubin, D.B. (1977), "Assignment to treatment group on the basis of a covariate", Journal of Educational and Behavioral Statistics, vol. 2, № 1.

Tsiatis, A. (2006), Semiparametric Theory and Missing Data, Nueva York, Springer. 


\section{Anexo A1}

En esta sección se presenta un breve resumen de los programas analizados en este artículo.

\section{a) Ley de Promoción de la Industria del Software}

En 2004, el Congreso de la Nación argentino aprobó la Ley de Promoción de la Industria del Software (Ley $N^{\circ}$ 25.922). En el marco de esta ley se redujeron los impuestos que han de pagar las empresas de software y servicios informáticos y se creó un fondo fiduciario llamado FONSOFT (presentado más adelante). Para recibir los beneficios fiscales que establece la ley, la empresa debe tener como principal actividad la creación, el diseño, el desarrollo y la producción e implementación de sistemas de software y su documentación técnica asociada. El software debe ser desarrollado íntegramente en Argentina.

Asimismo, las empresas deben satisfacer dos de los siguientes tres requisitos: i) incurrir en gastos propios de I+D; ii) disponer de certificaciones de calidad, y iii) realizar exportaciones.

Los beneficios fiscales consisten en:

i) Estabilidad fiscal durante la promoción del régimen (finales de 2019). De acuerdo con esto, las autoridades no pueden modificar el esquema de impuestos al sector de las empresas registradas en el régimen de promoción.

ii) Reducción implícita de las cargas de seguridad social. El 70\% de estos cargos puede acreditarse en el pago de otros impuestos nacionales (con excepción del impuesto a las ganancias).

iii) Reducción de hasta el 60\% en el monto a pagar por Impuesto a las Ganancias.

\section{b) FONSOFT}

Como se mencionó anteriormente, el Fondo Fiduciario de Promoción de la Industria del Software (FONSOFT) fue creado en 2004 en el marco de la Ley de Promoción de la Industria del Software. El FONSOFT es un programa que financia gastos de I+D en empresas del sector y la fase inicial de empresas que ingresan a la actividad mediante subsidios y créditos. El programa está involucrado en todas las etapas del proyecto innovador, desde su formulación e implementación hasta la evaluación de sus resultados. Solo pueden acceder a este financiamiento las pequeñas y medianas empresas (pymes) dedicadas a la producción de bienes y servicios de software.

EI FONSOFT está organizado en torno a cuatro instrumentos: i) subsidios a empresas para mejorar sus estándares de calidad o gastos de I+D; ii) créditos a empresas para promocionar el inicio o la consolidación de su actividad exportadora; iii) subsidios a empresarios orientados a la organización de nuevas empresas, y iv) subvenciones de contrapartida para financiar la capacitación de recursos humanos.

\section{c) FONTAR}

El Fondo Tecnológico Argentino (FONTAR) es otro de los fondos administrados por la Agencia Nacional de Promoción Científica y Tecnológica (ANPCyT) ${ }^{5}$. Los beneficiarios del FONTAR son

\footnotetext{
5 La Agencia Nacional de Promoción Científica y Tecnológica del Ministerio de Ciencia, Tecnología e Innovación Productiva se creó en 1996 con el objetivo de promover y apoyar las actividades científicas y tecnológicas.
} 
empresas innovadoras (en particular pymes) y organismos públicos que brindan asistencia técnica al sector privado. El financiamiento cubre la ejecución de proyectos innovadores por las siguientes vías: i) promoción de la ejecución de proyectos de I+D; ii) apoyo técnico a las empresas interesadas en formular proyectos; iii) asesoramiento técnico y financiero a las empresas que requieran financiamiento; iv) financiación de los proyectos seleccionados, y iv) concesión, en el marco de la Ley №23877, de un certificado de conformidad para los equipos o departamentos de I+D.

El programa se involucra en todas las etapas del proyecto innovador, desde su formulación e implementación hasta la evaluación de sus resultados. El FONTAR está organizado en componentes, subcomponentes e instrumentos de promoción. En la práctica, las empresas solicitan un instrumento en particular. Cada uno de ellos tiene criterios de selección propios y brinda apoyo en diferentes etapas del proceso innovador.

El FONTAR comprende un componente de innovación tecnológica en el sector productivo y un componente de Proyectos integrados de Aglomerados Productivos (PITEC). El primero se divide a su vez en tres subcomponentes que incluyen: i) proyectos de modernización tecnológica financiados con subsidios y préstamos; ii) apoyo al desarrollo tecnológico de pymes mediante subsidios (incluye la preparación de solicitudes de patentes), y iii) creación de capacidades para la provisión de servicios al sector productivo mediante préstamos reembolsables a organizaciones sin fines de lucro e instituciones privadas. 